\title{
Elaboración de perfiles y personalización de ofertas y precios en la contratación con consumidores
}

Profiling and price and offer personalization in consumer contracts

\author{
Antoni Rubí Puig \\ Profesor agregado de Derecho civil \\ Universitat Pompeu Fabra \\ E-mail: antoni.rubi-puig@upf.edu
}

\begin{abstract}
Resumen: La posibilidad de tratar grandes cantidades de datos (big data) y el desarrollo de algoritmos basados en inteligencia artificial y de nuevas herramientas de análisis han facilitado la personalización de diferentes elementos en la contratación online con consumidores. Mediante la elaboración de perfiles y otros tratamientos de datos personales, los empresarios consiguen personalizar sus ofertas, información, precios y servicios para consumidores individuales o segmentos de consumidores y, con ello, satisfacen mejor las preferencias de sus clientes. Sin embargo, los empresarios pueden recurrir a la personalización de sus contratos para apropiarse del excedente contractual que correspondería a los consumidores en un escenario uniforme, así como para manipular las preferencias de estos. El régimen jurídico previsto actualmente en el Reglamento General de Protección de Datos resulta insuficiente para dar respuesta a estos y otros riesgos que entraña la personalización.
\end{abstract}

Palabras clave: personalización, elaboración de perfiles, protección de datos personales, contratos con consumidores, discriminación de precios 


\begin{abstract}
The possibility of processing large datasets and the development of AI-based algorithms and new analytical tools have smoothed the personalization of online consumer contracts. Through profiling and other processing of personal data, businesses manage to personalize their offers, information, prices and services for individual consumers or for consumer segments, and with that they can better meet their customers' preferences. However, businesses may resort to personalization with the aims of appropriating the contractual surplus that would belong to consumers in a uniform scenario, and of manipulating their preferences as well. The legal regime currently provided for in the General Data Protection Regulation is insufficient to deal with these and other risks involved in personalization.
\end{abstract}

Keywords: personalization, profiling, data protection, consumer contracts, price discrimination

Sumario: 1. Introducción. 2. Personalización en la contratación online con consumidores. 3. Discriminación de precios y excedente contractual. 4. Tratamientos de datos personales para la elaboración de perfiles de consumidores. 4.1. Obtención. 4.2. Evaluación y análisis de los datos. 4.3. Combinación e intercambio de datos. 5. Protección de datos personales y personalización en la contratación online con consumidores. 5.1. General. 5.2. Régimen ad hoc sobre decisiones automatizadas y elaboración de perfiles. 5.3. Información y transparencia. 5.4. Derechos de los individuos. 6. Conclusiones. 7. Bibliografía.

\title{
1. Introducción
}

Las mejoras en las capacidades tecnológicas para tratar datos personales en masa han transformado la comercialización de bienes y la prestación de servicios en internet de un modo profundo. La posibilidad de tratar grandes cantidades de datos (big data) y el desarrollo de herramientas de inteligencia artificial aplicada, que permiten un alto grado de automatización y de autonomía, han facilitado a los empresarios un mayor conocimiento acerca de los consumidores, quienes, después de ser segmentados y vinculados a ciertos perfiles, reciben información, ofertas e incluso precios personalizados con arreglo a sus características personales observadas o inferidas.

La personalización en la prestación de servicios y comercialización de bienes ha conllevado importantes beneficios para usuarios y consumidores. Pero no todo son ventajas: son muchas las consecuencias negativas que pueden seguir a la elaboración de 
perfiles y a la adopción de decisiones de personalización y afectar a los consumidores y al bienestar social en su conjunto. Intromisiones ilegítimas en los derechos a la intimidad y a la protección de los datos personales (PASQUALE, F., 2016), discriminación en el acceso a bienes y servicios (KLEINBERG, J. - LUDWIG, S., et al, 2018), la pérdida del excedente contractual esperable (WAGNER, G. - EIDENMÜLLER, H., 2019), la manipulación de las preferencias (CALO, R., 2014), errores en la atribución de uno u otro perfil (CITRON, D. - PASQUALE, F., 2014), la alteración del comportamiento observable (chilling effects) (BÜCHI, M. - FOSCH VILLARONGA, E., et al, 2019) o el aislamiento social en la información recibida (information bubbles) (ZUIDERVEEN BORGESIUS, F. - TRILLING, D. et al, 2016) son algunos de los efectos negativos de la personalización en el ámbito de la contratación en masa con consumidores.

Por supuesto, mitigar o resolver cada uno de estos problemas requiere de soluciones diferentes y no necesariamente jurídicas. Junto a estas últimas no debería desdeñarse el papel que podrían desplegar la tecnología y el mercado para obtener lo mejor de la personalización y reducir a la vez su impacto negativo. En este trabajo nos preocupamos, sin embargo, de las soluciones jurídicas y solo desde el ámbito del derecho de protección de datos personales. Por ello, el análisis será inevitablemente parcial e insuficiente.

El trabajo se estructura del modo siguiente: el apartado 2 describe la personalización de diferentes elementos presentes en la contratación online con consumidores. El apartado 3 analiza la cuestión como un problema de maximización de beneficios por parte de empresarios: la personalización es sobre todo personalización de precios y aquellos persiguen identificar la predisposición de pago de cada consumidor y, con ello, extraer el excedente económico que obtendría el consumidor de contratar en un mundo sin personalización. El apartado 4 describe los tratamientos de datos personales que permiten la elaboración de perfiles de consumidores y su segmentación para una contratación personalizada. El apartado 5 examina el régimen jurídico vigente en materia de protección de datos personales aplicable a la personalización de consumidores y cuestiona su eficacia. Finalmente, el apartado 6 apunta unas breves conclusiones. 


\section{Personalización en la contratación online con consumidores}

Las tecnologías informáticas aplicadas a la contratación online han permitido aprovechar los datos personales de los consumidores y usuarios en línea y beneficiarse de la información y el conocimiento que aquellos generan. A partir de estos datos personales los empresarios han podido ajustar mejor los servicios que prestan y sus ofertas a sus clientes actuales o potenciales. Con ello, desaparece progresivamente uno de los rasgos que ha caracterizado la contratación en masa con consumidores desde hace tres cuartos de siglo: la uniformidad y fungibilidad en los tratos (sobre las transformaciones generales que han supuesto el uso masivo de datos personales para la contratación online, vid. LEVMORE, S. - FAGAN, F., 2018). De modo creciente, los consumidores van recibiendo un trato más personalizado: la información que pueden ver al visitar una tienda online, la publicidad y otras comunicaciones comerciales que reciben, las ofertas que les dirigen y, en su caso, los contratos a los que se adhieren y los servicios que les prestan difieren de los que reciben otros consumidores.

La personalización en los contratos con consumidores no es un fenómeno nuevo, ni tampoco exclusivo de un entorno en línea, pero ha sido en este ámbito y gracias a las operaciones de tratamiento masivo de datos personales y a los avances en los instrumentos de análisis sobre estos que ha adquirido un mayor protagonismo y sofisticación.

Hay muchas estrategias y modalidades diversas de personalización en la contratación con consumidores online. La información disponible sobre experiencias, tanto exitosas como controvertidas, es muy nutrida. Por ejemplo, un consumidor puede tener que pagar un precio para acceder al mismo bien diferente al que debe satisfacer otro consumidor en el mismo sitio web.

\footnotetext{
Si bien es habitual que las condiciones de financiación o de crédito al consumo sean personalizadas en varios tipos de bienes adquiridos por consumidores, la elaboración de perfiles y el uso de herramientas analíticas permiten trasladar estas prácticas a más ámbitos y una mayor automatización. En previsión de los riesgos que puede entrañar la personalización en el análisis de la solvencia del consumidor y en la determinación de las condiciones de acceso al crédito, la reciente Propuesta de Directiva relativa a los créditos al consumo de 30.6.2021(COM (2021) 347 final) incluye normas sobre transparencia informativa y restricciones.
} 
La interfaz de usuario del sitio web donde realizar una compra puede ser también diferente en función de las características personales del consumidor. La información que se le muestre inicialmente también podrá diferir de la que vean otros usuarios: por ejemplo, al acceder al sitio web podrá ver productos que estén de alguna forma relacionados con el historial de consumo del usuario o de las características inferidas por el vendedor a partir de la observación del comportamiento de aquel.

Esta es una de las preocupaciones que subyace a las Propuestas de 15.12.2020 de Reglamento sobre mercados disputables y equitativos en el sector digital (Ley de Mercados Digitales) y de Reglamento relativo a un mercado único de servicios digitales (Ley de servicios digitales).

Ello es especialmente relevante en plataformas de recomendación y también en sitios web que ofrecen servicios de información o puesta a disposición de contenidos (por ejemplo, los algoritmos de recomendación de YouTube o de Spotify). También la publicidad que recibe un usuario - tanto por el contenido de esta como, muy especialmente, por su forma y características de tiempo y lugar- también es objeto de personalización.

Además de la personalización de los elementos y aspectos de la contratación con consumidores, las decisiones automatizadas sobre perfiles permiten también controlar factores como el mejor momento para suministrar una información a un consumidor. Por ejemplo, a partir del estado de ánimo observado o inferido de un consumidor, un determinado mensaje publicitario enviado en uno u otro momento puede incrementar las probabilidades de que aquel individuo decida acceder a un bien o servicio. La tecnología permite a los empresarios no solo llegar a aquellos consumidores más receptivos, sino también hacerlo en el momento en que más lo estarán. En otros términos, la personalización de diferentes factores que inciden en la contratación online se optimiza a tiempo real (WILLIS, 2020, 121-122; VEALE, M. - ZUIDERVEEN BORGESIUS, F., 2021).

\section{Discriminación de precios y excedente contractual}

La personalización en la contratación con consumidores permite, frente a una contratación uniforme, que los empresarios incrementen el número de transacciones y, además, obtengan en ellas la mayoría de su excedente contractual. La personalización de los diferentes elementos del contrato -y particularmente, de los precios- les permite así obtener más beneficios y, precisamente, esta promesa de futuros ingresos les genera 
incentivos para apoderarse y controlar más datos personales y proceder a un perfilamiento más afinado de los consumidores (entre otros, ALFARO, J., 2017; BAR-GILL, O., 2017; MAGGIOLINO, M., 2017; ZUIDERVEEN BORGESIUS, F. - POORT, J., 2017).

La personalización es una forma más de discriminación de precios, un fenómeno habitual en muchos mercados tanto dentro como fuera de internet y que, en términos generales, puede definirse como la comercialización de unos determinados bienes y servicios en condiciones diferentes para diferentes grupos de consumidores, sin que ello responda a las diferencias en las características de los productos o servicios (VARIAN, H., 2014, 480). En el límite, un consumidor acaba satisfaciendo un precio diferente al que abona otro consumidor por una mercancía idéntica. Los economistas han distinguido diferentes formas de discriminación de precios. Entre ellas, han construido teóricamente la noción de discriminación de primer grado. Conforme a esta, un empresario podría estar en condiciones de identificar el precio al que cada consumidor individual estaría dispuesto a contratar y ofrecerle el bien a cambio de este precio individualizado. Las operaciones de big data contribuyen progresivamente a un conocimiento más cabal de la predisposición a pagar de cada consumidor y aproximan el constructo teórico de la discriminación de primer grado a una realidad práctica.

\footnotetext{
Sobre las diferentes formas de discriminación de precios, RUBÍ PUIG, A., 2015, 29-37 y bibliografía que allí se cita. En particular, las formas más habituales hasta la fecha han sido la discriminación de segundo grado y la de tercer grado. La primera hace referencia a supuestos en los cuales un empresario crea los mecanismos para que los consumidores revelen su predisposición a pagar por medio de sus decisiones de compra, por ejemplo, prefiriendo adquirir un libro de tapa dura y no esperar a que esté disponible la versión en rústica o de bolsillo. En la discriminación de tercer grado, el empresario agrupa a los consumidores en conjuntos más o menos homogéneos que se correspondan aproximadamente con su capacidad o predisposición a pagar por acceder a los bienes o servicios. Por ejemplo, un comerciante de software ofrece precios diferentes para usuarios profesionales y para estudiantes.
}

Desde el punto de vista del bienestar económico, la discriminación de precios presenta un beneficio importante frente a un sistema de precios uniformes. Consumidores que estarían dispuestos a satisfacer un precio superior al coste de producir el bien o prestar el servicio pero inferior al precio uniforme que un empresario con poder de mercado utilizaría y que, por tanto, no podrían adquirir o acceder a aquel, podrán hacerlo gracias a la personalización de precios.

Imaginemos un mercado hipotético en el que un empresario X comercializa una determinada mercancía cuyo coste de producción por unidad es de 3 u. Hay en ese mercado tres consumidores potenciales que 
valoran de forma diferente la mercancía y tienen una predisposición a adquirirla divergente. Así, el consumidor A valora el bien de forma que estaría dispuesto a pagar 8 u por él, el consumidor B, 6 u y el consumidor C, 4 u. El empresario puede fijar un precio uniforme por el bien. Así si lo vende por 4 u, podrá vender 3 unidades y obtener un beneficio final de $3 \mathrm{u}$. Si lo vende por 5 u, venderá solo 2 unidades, pero su beneficio será de $4 \mathrm{u}$. Si lo vende por $6 \mathrm{u}$, venderá 2 unidades con un beneficio de 6 u. Si vende por $7 \mathrm{u}$, venderá solo 1 unidad con un beneficio de $4 \mathrm{u}$. Finalmente, si vende por $8 \mathrm{u}$, también venderá 1 unidad con un beneficio de $5 \mathrm{u}$. Teniendo en cuenta esta información, el empresario, para maximizar sus beneficios, preferirá fijar un precio uniforme de $6 \mathrm{u}$. En consecuencia, el consumidor $\mathrm{C}$ no podrá adquirir el bien. Si fuera posible discriminar en precios de forma efectiva y ajustar un precio personalizado para cada consumidor equivalente a su predisposición, entonces, los tres consumidores sí podrían adquirir el bien: A pagaría 8 u por él, B pagaría $6 \mathrm{u}$, y C pagaría 4 u. Los beneficios del empresario X serían 9 u. (equivalentes a (8-3)+(6-3)+(4-3)).

Este resultado comporta la reducción de la ineficiencia estática derivada del establecimiento de precios por parte de operadores con poder de mercado y que es conocida como pérdida de excedente o peso muerto (deadweight loss). La discriminación de precios permite recuperar estas rentas que se perdían para la sociedad, aunque el beneficiario último de estas será el empresario. Mas el empresario no únicamente se apropia de este excedente, sino también del que obtendrían los demás consumidores en un mercado con precios fijados uniformemente.

En nuestro ejemplo, el consumidor A obtenía un excedente de $2 \mathrm{u}$ al adquirir el producto: su valoración de este era de 8 u y lo adquiría por 6 u. Con un sistema de discriminación de precios, este excedente del consumidor A es apropiado por el empresario.

Desde la óptica de la eficiencia económica, la reducción de la deadweight loss constituye un importante argumento en favor de la discriminación de precios. Además, el ordenamiento jurídico cuenta con herramientas, como el sistema tributario, que permiten cierta distribución ex post del excedente apropiado por los empresarios.

También unos mayores ingresos pueden ser invertidos en investigación para reducir el coste de producción y, si hay cierta competencia o un riesgo de competencia futura, inducir a los empresarios a una personalización diferente de precios, que permitiera cierta apropiación del excedente por los consumidores.

También podría argüirse que si un individuo adquiere un bien por el precio al que realmente está dispuesto a satisfacer no debería preocupar que no pueda obtener un excedente o que otros satisfagan precios diferentes (vid. la parábola de los jornaleros en Mateo 20, 1-16). Sin embargo, en la realidad, los individuos por lo general perciben muchas formas de discriminación de precios como injustas e ilegítimas (MILLER, A., 2014). Este rechazo moral comporta que, en la práctica, la discriminación de precios haya de fundarse en una determinada justificación o realizarse en situaciones en las cuales sea 
muy difícil de observar por parte de consumidores y usuarios. Así, por ejemplo, estrategias de discriminación de precios consistentes en descuentos para estudiantes, para jóvenes o para usuarios frecuentes no suelen plantear ningún reproche. En cambio, saber que un consumidor acaba pagando un precio superior solo porque tiene un poder adquisitivo superior o está en una situación transitoria que le lleva a valorar más el bien no despierta demasiadas simpatías. Por ello, los empresarios suelen recurrir a estas formas de personalización únicamente si pueden desplegarse de un modo invisible (BAR-GILL, O., 2017, 227-228). Este puede ser el caso, por ejemplo, de transacciones en las cuales los consumidores tengan dificultades para contrastar sus condiciones con las de otros sujetos; o en las cuales sea difícil recurrir al arbitraje (esto es, la adquisición de los bienes directamente de otros compradores); o cuando los precios y bienes fluctúan por otros motivos, lo que dificulta comprender cuáles son los factores que acaban determinando el precio final a satisfacer por cada consumidor o usuario (ej.: billetes de avión).

La personalización de las ofertas y de la información que reciben los consumidores especialmente, a partir de comunicaciones comerciales personalizadas- puede utilizarse no solo para conocer la predisposición a pagar de los consumidores, sino también para manipularla (BAR-GILL, O., 2017, 237-242). En estos casos, es mucho más dudoso que la discriminación de precios sea socialmente eficiente, pues la recuperación de la deadweight loss no necesariamente va a compensar la pérdida de rentas que sufren aquellos consumidores que no habrían adquirido el bien si su predisposición a pagar no hubiera sido manipulada.

\section{Tratamientos de datos personales para la elaboración de perfiles de consumidores}

La personalización en los contratos con consumidores es resultado, en gran medida, de varias operaciones de tratamiento sobre datos personales. La metáfora de los datos personales como el nuevo petróleo está ya muy gastada, pero sirve todavía para señalar cómo son aquellos los que nutren y hacen funcionar, con provecho, los sistemas de personalización que implementan los empresarios en sus actividades económicas. Para ofrecer unas condiciones cada vez más personalizadas y ajustadas a las preferencias, necesidades, capacidades y, en su caso, a las vulnerabilidades de sus clientes y maximizar con ello sus beneficios, los empresarios perseguirán obtener el mayor número de datos personales, analizarlos, combinarlos con otros y utilizarlos para la adopción de 
determinadas decisiones que afectarán a los consumidores (para un análisis exhaustivo de las diferentes operaciones de tratamiento y de los agentes implicados en ellas, SARTOR, G. - LAGIOIA, F. - GALLI, F., 2021).

\subsection{Obtención}

Son varias las fuentes de obtención de datos personales de los consumidores. Un primer grupo de datos provendrá de información suministrada por los propios individuos, por ejemplo, al rellenar un formulario de alta de un servicio o al subir determinados contenidos a una red social. Un segundo grupo de datos será directamente observado por el empresario o un tercero, por ejemplo, a partir del uso concreto que el individuo realice de un sitio web o del historial de consumo de un determinado consumidor. Finalmente, un tercer grupo de datos personales puede ser obtenido a partir de la evaluación y análisis de varios datos (infra 4.3). Por ejemplo, la atribución de un determinado perfil o segmento a partir de las inferencias realizadas sobre un sujeto puede constituir un nuevo dato personal si se utiliza para adoptar una determinada decisión que le incumba, por ejemplo, la personalización de la oferta que se le dirige.

La tipología de los datos personales que pueden obtenerse es muy diversa. Entre la información obtenida, pueden destacarse, entre otros, datos de contacto, datos sociodemográficos tales como la edad, género, nivel educativo, sexualidad, religión, ocupación e ingresos, datos de geolocalización, datos técnicos sobre el dispositivo con el cual se accede a una determinada página web, datos de comportamiento, historial de navegación, estados de ánimo y emociones, o valores personales (sobre la tipología de datos personales empleados en la personalización de precios, MILLER, A., 2014, 49-54). A mayor número de datos y a mayor complejidad y granularidad, más probabilidades de que la personalización sea más efectiva.

Los niveles de transparencia en la obtención de datos personales pueden ser también muy diferentes. Especialmente, en relación con los datos observados por un responsable del tratamiento, hay muchas tecnologías que permiten obtener datos de usuarios sin que estos sean mínimamente conscientes de ello y de su uso posterior. Muchos de estos datos no identifican directamente a un individuo, pero, en combinación con otros, sí son susceptibles de relacionarse con una persona y de utilizarse para adoptar decisiones que 
le puedan afectar. Estos datos pueden ser obtenidos por medio de cookies de navegador, pero también por huellas digitales, web beacons y otras tecnologías. En ocasiones, los responsables del tratamiento se aprovechan de sesgos cognitivos de los usuarios y contextualizan la información o el marco en el cual aquellos han de tomar una decisión para que sean más proclives a proporcionar más datos personales. Estas estrategias se conocen popularmente como dark patterns (WILLIS, L., 2020, 132-150).

\subsection{Evaluación y análisis de los datos}

Las operaciones de evaluación y análisis constituyen en sí mismas tratamientos de datos personales. Sirven, en buena medida, para identificar patrones generales de comportamiento y correlaciones entre determinados rasgos y decisiones individuales. A partir del análisis de los datos y de los modelos desarrollados, se persigue elaborar perfiles únicos de usuarios para la posterior adopción de decisiones de personalización. La atribución de un perfil determinado constituye casi siempre un nuevo dato personal. En los últimos años, las herramientas técnicas que se han desarrollado para el análisis de datos personales se han sofisticado significativamente y han aumentado los niveles de fiabilidad de las inferencias y predicciones. Pueden destacarse sobre todo los análisis masivos de datos (big data analytics) y el desarrollo de herramientas de inteligencia artificial, con ciertas capacidades de autonomía y autoaprendizaje (machine learning). Estas herramientas de evaluación y análisis estarán las más de las veces protegidas por derechos de propiedad intelectual y secretos empresariales.

\subsection{Combinación e intercambio de datos}

Finalmente, la elaboración de perfiles y la adopción de decisiones de personalización implica también la combinación e intercambio de las bases de datos propias con datos personales suministrados por otros, tales como intermediarios o prestadores de servicios. Toda una industria ha emergido en torno a la explotación económica de los datos personales. Hay compañías especializadas, denominadas habitualmente brokers, que construyen bases de datos personales obtenidos a partir de fuentes privadas y públicas, y que comercializan. También hay empresas especializadas en prestar servicios de análisis y evaluación, que utilizan sus propias bases de datos en combinación con las de sus 
clientes para asesorarles en la adopción de decisiones, por ejemplo, en el ámbito del marketing online (VEALE, M. - ZUIDERVEEN BORGESIUS, F., 2021).

La pluralidad de sujetos implicados en las tareas de personalización de los contratos de consumo complica la atribución de responsabilidades y la calificación de las relaciones jurídicas entre ellos. Los problemas derivarán en muchos casos de la dificultad de determinar si una operación de tratamiento se realiza conjuntamente por varios corresponsables o si uno de los sujetos asume la posición de un encargado del tratamiento en nombre ajeno.

\section{Protección de datos personales y personalización en la contratación online con consumidores}

\subsection{General}

El Reglamento General de Protección de Datos constituye la principal normativa de aplicación a los tratamientos de datos implicados en las operaciones necesarias para la elaboración de perfiles y la adopción de decisiones de personalización que afectan a la contratación en línea con consumidores ${ }^{1}$.

El art. 4.4 RGPD define la "elaboración de perfiles" como "toda forma de tratamiento automatizado de datos personales consistente en utilizar datos personales para evaluar determinados aspectos personales de una persona física, en particular para analizar o predecir aspectos relativos al rendimiento profesional, situación económica, salud, preferencias personales, intereses, fiabilidad, comportamiento, ubicación o movimientos

\footnotetext{
${ }^{1}$ Reglamento (UE) 2016/679 del Parlamento Europeo y del Consejo de 27 de abril de 2016 relativo a la protección de las personas físicas en lo que respecta al tratamiento de datos personales y a la libre circulación de estos datos y por el que se deroga la Directiva 95/46/CE (Reglamento general de protección de datos) (DOUE L 119, de 4.5.2016, 1-88) (en adelante, "RGPD"). Ténganse en cuenta también, en el derecho español, la Ley Orgánica 3/2018, de 5 de diciembre, de Protección de Datos Personales y garantía de los derechos digitales, y la Ley 34/2002, de 11 de julio, de servicios de la sociedad de la información y de comercio electrónico (que transpone al derecho español la Directiva de e-Privacidad, especialmente en todo lo relativo a las cookies de seguimiento (Directiva 2002/58/CE del Parlamento Europeo y del Consejo, de 12 de julio de 2002, relativa al tratamiento de los datos personales y a la protección de la intimidad en el sector de las comunicaciones electrónicas)). La Propuesta de Reglamento de e-Privacidad de 10 de febrero de 2021 (2017/0003(COD)) contiene algunas reglas específicas que, de aprobarse, restringirán más la elaboración de perfiles.
} 
de dicha persona física”. En efecto, la elaboración de perfiles debe realizarse de forma, al menos parcialmente, automatizada; debe servirse de datos personales; y debe tener como finalidad la evaluación de determinados aspectos de un individuo. Es este último aspecto el más relevante y el que sirve para distinguir la elaboración de perfiles de otras formas de tratamiento que persiguen también utilizar determinados aspectos personales pero no con una finalidad de evaluación individualizada. Por ejemplo, una simple clasificación de las personas basada en características conocidas como su edad, sexo y altura no da lugar necesariamente a una elaboración de perfil.

Así, si una empresa clasifica a sus clientes según su edad o género con motivos estadísticos y para obtener una visión global de estos sin hacer luego predicciones ni evaluar a una persona, el tratamiento de datos no consistiría en una elaboración de un perfil y, en consecuencia, no serían de aplicación las especificidades previstas para este ámbito.

La evaluación de un individuo persigue poder realizar deducciones estadísticas, predicciones, correlaciones o análisis, en definitiva, juicios de valor, en relación con un sujeto determinado. En función de esta evaluación, que puede ser más o menos automatizada, el responsable puede adoptar luego decisiones sobre el individuo, que también pueden ser más o menos automatizadas, entre las cuales, las relativas a la personalización en el ámbito de los contratos con consumidores.

El art. 22 RGPD prevé un régimen jurídico ad hoc para los tratamientos relacionados con la elaboración de perfiles y la adopción de decisiones automatizadas. Sin embargo, las condiciones de aplicación de este régimen comportan que el precepto tenga un alcance más bien limitado. Por ello, gran parte de las operaciones de tratamiento de datos personales implicadas en la elaboración de perfiles y la personalización no estarán cubiertas por el art. 22 RGPD y se regirán por el régimen general.

Excede de este trabajo un examen pormenorizado de todo el régimen jurídico general previsto en el RGPD y aplicable a la personalización en el ámbito de los contratos con consumidores. Por ejemplo, la especificación de las obligaciones que incumben a responsables del tratamiento, tales como el deber de realizar una evaluación de impacto, o la concreción de los principios de responsabilidad activa o de minimización de los datos dependerán mucho de las características técnicas de la operación de tratamiento a realizar; así como también la implicación de encargados del tratamiento o de corresponsables. Por ello, este trabajo presenta solo algunas valoraciones generales acerca de los derechos de los individuos en este ámbito y sobre las reglas específicas previstas en el art. 22 RGPD.

\subsection{Régimen ad hoc sobre decisiones automatizadas y elaboración de perfiles}


El art. 22 RGPD establece, desde la perspectiva de los individuos titulares de datos personales, un conjunto de derechos en relación con decisiones individuales automatizadas, incluida la elaboración de perfiles, que les conciernen (GRUPO DE TRABAJO SOBRE PROTECCIÓN DE DATOS DEL ARTÍCULO 29, 2017; VILASAU, M., 2020). El punto de partida es una prohibición de estas formas de tratamiento. Así, el art. 22.1 RGPD establece que "[t]odo interesado tendrá derecho a no ser objeto de una decisión basada únicamente en el tratamiento automatizado, incluida la elaboración de perfiles, que produzca efectos jurídicos en él o le afecte significativamente de modo similar". Ahora bien, dos factores acotan el alcance de esta prohibición general. Por un lado, el art. 22 RGPD posee un ámbito de aplicación muy limitado. Y, por otro, son varias las circunstancias en las cuales esta prohibición general decae y son posibles estas formas de tratamiento si van acompañadas de determinadas garantías adicionales.

Ámbito de aplicación limitado: las condiciones de aplicación del art. 22 RGPD determinan que esta sea más bien limitada. Así el precepto exige que: a) la decisión que afecte a un individuo se base "únicamente" en un tratamiento automatizado de sus datos personales; y que b) la decisión produzca efectos jurídicos en el individuo o le afecte significativamente de un modo similar.

El hecho de que el tratamiento de los datos no esté exclusivamente automatizado ya excluye la aplicación del art. 22 RGPD. Por ello, un responsable puede fácilmente eludir sus obligaciones mediante la previsión de una intervención humana en algún paso (human in the loop). Para evitar este resultado, varios autores han propuesto una interpretación restrictiva: no bastaría con que alguien supervisara o verificara las decisiones adoptadas por el algoritmo, sino que este sujeto debería tener control y poder suficiente para alterar el signo de la decisión adoptada. También sería cuestionable excluir la aplicación del art. 22 RGPD en supuestos de elaboración de perfiles resultantes de la integración de diversos pasos o fases, si algunas y solo algunas de estas cumplen con todos los requisitos previstos en el precepto (BINNS, R. - VEALE, M., 2021).

Por otra parte, la determinación de qué tipo de decisiones quedan cubiertas por el precepto también plantea problemas. En primer lugar, el art. 22 no proporciona una definición de “decisión” y, por lo tanto, puede cuestionarse si todas las modalidades de personalización 
constituyen decisiones en el sentido del precepto. En segundo lugar, la decisión ha de producir efectos jurídicos en el interesado o ha de afectarle significativamente de modo similar. Es dudoso que muchas de las decisiones de personalización en el ámbito de los contratos con consumidores produzcan efectos jurídicos en el sentido del precepto. Por ejemplo, si bien la emisión de una oferta produce efectos jurídicos, estos alcanzan básicamente la esfera del ofertante y no la del destinatario. En otros términos, la emisión de una oferta personalizada no supone un cambio en la situación jurídica del destinatario, ni le genera nuevos derechos u obligaciones (como ocurriría, por ejemplo, en el caso de una decisión automatizada que comportara una terminación contractual). En efecto, a falta de efectos jurídicos, será preciso contemplar si una decisión de personalización puede tener efectos significativamente similares. En general, muchas decisiones automatizadas basadas en perfiles personales tendrán estos efectos. Así, por ejemplo, si, a partir de la elaboración de un perfil, una entidad de crédito deniega un préstamo a un sujeto, muy probablemente esta decisión afectará significativamente a esa persona de modo similar a que si tuviera efectos jurídicos.

En este examen, se exige, en general, que una decisión tenga el potencial de afectar significativamente a las circunstancias, al comportamiento o a las elecciones de las personas afectadas; de tener un impacto prolongado o permanente en el interesado; o, en los casos más extremos, de provocar la exclusión o discriminación de personas.

Ejemplos de ello incluirían decisiones que afectaren a las circunstancias financieras de una persona, como su elegibilidad para un crédito; decisiones que afectaren al acceso de una persona a los servicios sanitarios; decisiones que denegaren a una persona una oportunidad laboral o que la colocaren en gran desventaja; o decisiones que afectaren al acceso de una persona a la educación, por ejemplo, su ingreso en la universidad.

Desde este punto de vista, es difícil entender que las decisiones de personalización en los contratos de consumo puedan afectar a un individuo de un modo significativo. El hecho de que se le muestre una determinada información en un sitio web, que se le envíe una determinada comunicación comercial, que se le muestre un precio -por ejemplo, cincuenta céntimos superior al que le muestra a su vecino-, o que le recomienden la adquisición de otros productos relacionados difícilmente tendrá un impacto sustancial en el consumidor individual. Ahora bien, si las decisiones individualizadas de personalización afectan a todos los miembros de un determinado grupo social (por ejemplo, un grupo étnico o lingüístico) y se agregan los diferentes impactos el efecto 
global puede devenir significativo. Varios estudios empíricos han mostrado cómo decisiones de personalización pueden perpetuar estereotipos sociales y llevar a la discriminación.

Uno de los primeros estudios mostraba cómo ciudadanos negros en EE.UU. recibían muchos más anuncios sobre servicios de supresión de antecedentes penales que ciudadanos blancos (SWEENEY, L., 2013).

Acaso el ordenamiento jurídico disponga de otras herramientas para combatir estos problemas, pero el derecho de la protección de datos personales no tiene una lógica únicamente individual. Despliega también una dimensión colectiva a la cual contribuyen factores como el impacto que tiene el suministro de datos por una persona para otras personas con las que guarda cierta afinidad o comparte grupo social (WACHTER, S., 2020) y (BAROCAS, S. - LEVY, K., 2020). Hay, pues, una salvaguarda de intereses generales, especialmente en supuestos como estos, en los cuales, un problema de acción colectiva impide una aplicación eficaz del derecho (RUBÍ PUIG, A., 2019, 206-213).

Excepciones a la prohibición: el derecho a no ser objeto de una decisión basada únicamente en el tratamiento automatizado, incluida la elaboración de perfiles, queda excluido en tres situaciones (art. 22.2 RGPD). En otros términos, el RGPD permite la adopción de decisiones o la elaboración de perfiles completamente automatizadas si ello: a) es necesario para la celebración o la ejecución de un contrato entre el interesado y un responsable del tratamiento; b) está autorizado por el Derecho de la Unión o de los Estados miembros que se aplique al responsable del tratamiento y que establezca asimismo medidas adecuadas para salvaguardar los derechos y libertades y los intereses legítimos del interesado; o c) se basa en el consentimiento explícito del interesado ${ }^{2}$. En consecuencia, muchas modalidades de personalización en los contratos con consumidores estarán legitimadas o bien por el consentimiento de estos últimos o por resultar necesarias en relación con la ejecución del contrato.

Para estos dos últimos casos, el RGPD prevé la obligación del responsable del tratamiento de establecer determinadas garantías adicionales para proteger a los titulares de datos personales. En particular, el art. 22.3 RGPD obliga a la adopción de medidas adecuadas

\footnotetext{
${ }^{2}$ Véase también art. 22.4 RGPD, que permite el tratamiento de categorías especiales de datos personales si hay consentimiento explícito del interesado conforme al art. 9.2 a RGPD.
} 
para salvaguardar los derechos y libertades y los intereses legítimos del interesado, que incluyan como mínimo el derecho a obtener intervención humana por parte del responsable, a expresar su punto de vista y a impugnar la decisión.

\begin{abstract}
Para reforzar la protección de los datos personales, (GRUPO DE TRABAJO SOBRE PROTECCIÓN DE DATOS DEL ARTÍCULO 29, 2017, 34-37) incluye una serie de recomendaciones de buenas prácticas para mitigar los problemas derivados de la elaboración de perfiles y la automatización de las decisiones que afectan a los individuos. Estas recomendaciones también se dirigen a operaciones de tratamiento no cubiertas por el art. 22 RGPD. Entre las medidas recomendadas, pueden destacarse los controles periódicos de aseguramiento de la calidad de los sistemas; auditorías algorítmicas dirigidas a comprobar los algoritmos utilizados y desarrollados por los sistemas de aprendizaje automático para demostrar que funcionan según lo previsto, y que no producen resultados discriminatorios, erróneos o injustificados; auditorías independientes de terceros; conseguir garantías contractuales sobre algoritmos proporcionados por terceros; utilización de técnicas de anonimización y seudoanonimización; mecanismos de certificación para operaciones de tratamiento; códigos de conducta para procesos de auditoría que impliquen aprendizaje automático; o comités de ética para evaluar los daños y beneficios potenciales para la sociedad de aplicaciones concretas de la elaboración de perfiles.
\end{abstract}

Además, el RGPD refuerza los derechos de información y de acceso de los particulares en los supuestos en los cuales el art. 22 resulta de aplicación (infra 5.3 y 5.4).

\title{
5.3. Información y transparencia
}

La transparencia forma parte de uno de los principios generales que han de informar cualquier operación de tratamiento de datos personales (art. 5.1 a RGPD). El RGPD contempla en sus arts. 13 y 14 los deberes específicos de información que incumben a los responsables del tratamiento. Entre estos, destaca un refuerzo de la transparencia en aquellos supuestos cubiertos por el art. 22 RGPD: se establece una obligación del responsable de proporcionar "información significativa sobre la lógica aplicada, así como la importancia y las consecuencias previstas de dicho tratamiento para el interesado" (arts. 13.2 $f$ y $14.2 \mathrm{~g}$ RGPD)). Algunos autores se han referido a este plus de transparencia como un "derecho a una explicación" o un derecho a abrir la caja negra (blackbox) de los algoritmos (para las discusiones sobre el alcance de este derecho, CASEY, B. FARHANGI, A. - VOGL, R., 2019). Este derecho a obtener información sobre la lógica aplicada o su correlativo deber de transparencia están sujetos a varias limitaciones en la práctica. En primer lugar, puede ser difícil de cumplir en supuestos en los cuales la lógica del algoritmo va modificándose progresivamente y de forma autónoma (machine learning). En segundo lugar, se plantean dudas acerca del concepto de explicación y de las posibles modalidades de suministrarla: por ejemplo, la información a suministrar 
puede consistir en una explicación general del modelo (model-based explanation) o puede consistir en explicar cómo se adoptó una decisión basada en los datos particulares del afectado (subject-based explanation). En tercer lugar, los algoritmos empleados para la adopción de decisiones o la elaboración de perfiles estarán las más de las veces protegidos por derechos de propiedad intelectual y secretos empresariales, que deberán también ser respetados y ponderados con los intereses de los individuos afectados por el tratamiento de sus datos personales.

El hecho de que muchas decisiones de personalización en el ámbito de la contratación con consumidores no tengan un impacto significativo en estos comportará que este deber reforzado de transparencia no sea de aplicación y que únicamente incumban al responsable deberes generales de información. En este sentido, estará obligado a informar, entre otros extremos, del tipo de tratamiento llevo a cabo y de sus finalidades y, en definitiva, deberá informar sobre la elaboración de perfiles con finalidades de personalización ${ }^{3}$. Con todo, como es sabido, los deberes de transparencia informativa en el ámbito del derecho de consumo son, por lo general, poco efectivos (BEN-SHAHAR, O. - SCHNEIDER, C., 2014).

La falta de información puede menoscabar la presencia de un verdadero consentimiento efectivo y explícito a los tratamientos de datos implicados en la personalización de contratos y deteriorar la idea de autodeterminación informativa que subyace al RGPD.

\begin{abstract}
El consentimiento del interesado constituye la principal base de legitimación de un tratamiento de datos personales (art. 6.1 $a$ RGPD). En el ámbito de la personalización, también el consentimiento del consumidor legitima los tratamientos sobre sus datos necesarios para la personalización de sus elementos, pues la prestación de un servicio no necesariamente será una base suficiente para proceder a tratamientos de datos encaminados a una personalización (COMITÉ EUROPEO DE PROTECCIÓN DE DATOS, 2019, 14).
\end{abstract}

A pesar de que el RGPD exija la prestación de un consentimiento mediante un acto afirmativo claro que refleje una manifestación de voluntad libre, específica, informada, e inequívoca del interesado de aceptar el tratamiento de datos de carácter personal que le conciernen $^{4}$, son muchos los problemas que afectan a las maneras en las cuales este se

\footnotetext{
${ }^{3}$ Vid. también arts. 6.1 y 6 bis de la Directiva 2011/83/UE, sobre los derechos de los consumidores (después de su reforma por Directiva (UE) 2019/2161 del Parlamento Europeo y del Consejo de 27 de noviembre de 2019).
} 
obtiene online. La aceptación sin la lectura de la información relevante, las dificultades para comprenderla o para anticipar las posibles consecuencias de uno u otro tratamiento, la utilización de dark patterns, o el impulso para acceder rápidamente a los bienes o servicios de que se trate, dificultan que un consumidor pueda consentir con conocimiento suficiente a estar sujeto a decisiones de personalización. En especial, es dudoso que puede hablarse de un consentimiento prestado libremente a estas formas de tratamiento si el acceso a los bienes o la prestación de servicios se realiza necesariamente en condiciones personalizadas. No obstante, varias propuestas legales de reforma en el derecho de la UE se orientan en buena medida a reforzar la transparencia informativa y el consentimiento ${ }^{5}$.

\subsection{Derechos de los individuos}

El RGPD reconoce un conjunto de derechos de los individuos en relación con los tratamientos de datos personales que les incumben, pero que, en varios supuestos de personalización, pueden tener poco recorrido (WACHTER, S. - MITTELSTADT, 2019, 542-568) y (FINCK, M., 2021, 96). Así, derechos como el de acceso, rectificación, supresión u objeción no siempre serán efectivos para combatir problemas derivados de la personalización en los contratos de consumo.

En los supuestos de elaboración de perfiles y decisiones completamente automatizadas, el RGPD confiere a los particulares un derecho a obtener del responsable del tratamiento confirmación de si se están tratando o no datos personales que le conciernen y, en tal caso, derecho de acceso a los datos personales y a la información relativa a la existencia de decisiones automatizadas, incluida la elaboración de perfiles, e información significativa sobre la lógica aplicada, así como la importancia y las consecuencias previstas de dicho tratamiento para el interesado (art. 15.1 $h$ RGPD).

El principal problema de fondo proviene del incierto estatus jurídico de las inferencias, correlaciones, predicciones u opiniones utilizadas en el ámbito de la personalización (WACHTER, S. - MITTELSTADT, 2019). Como se ha señalado, mediante diversas herramientas los responsables del tratamiento de datos combinan datos personales de

\footnotetext{
4 Cdo 32 RGPD. Vid. también STJUE de 1 de octubre de 2019, C-673/17, Verbraucherzentrale Bundesverband eV c. Planet49 GmbH; y STJUE de 11 de noviembre de 2020, C-61/19, Orange Romania SA c. ANSPDCP.

${ }^{5}$ Por ejemplo, arts. 5 y 11 de la Propuesta de Reglamento del Parlamento Europeo y del Consejo sobre mercados disputables y equitativos en el sector digital (Ley de Mercados Digitales), de 15.12.2020 (COM(2020) 842 final); y arts. 24, 26 y 27 de la Propuesta de Reglamento del Parlamento Europeo y del Consejo relativo a un mercado único de servicios digitales (Ley de servicios digitales), de 15.12.2020 $(\mathrm{COM}(2020) 825$ final).
} 
diferentes fuentes y a partir de ellos obtienen cierta información que no necesariamente será cierta en relación con un determinado individuo. Por ejemplo, a partir de la fuerza con que un usuario teclea o de los filtros de color que utiliza en las fotografías que sube a una red social, un algoritmo puede predecir cuál es el estado de ánimo probable de aquél. El responsable puede utilizar luego esta inferencia -por ejemplo, es altamente probable que el usuario $\mathrm{X}$ esté triste- para dirigirle anuncios con un contenido personalizado para este segmento de consumidores, en determinadas franjas horarias o al visitar determinados contenidos online. Estas inferencias no dejan de ser juicios de valor que formula el responsable del tratamiento, a partir del uso de sus algoritmos de evaluación y otras herramientas. En tanto que opiniones, es cuestionable que puedan calificarse como datos personales. Pero, además, si se supera este primer escollo, su carácter subjetivo podría limitar el ejercicio de derechos de los afectados como el de rectificación.

En general, el RGPD parte de un concepto amplio de dato personal (art. 4.1 RGPD), que a su vez ha sido interpretado de forma amplia por las autoridades de supervisión y por los tribunales. Conforme a este concepto, una determinada información puede constituir un dato personal si se utiliza con una finalidad de evaluación o puede afectar a un individuo ${ }^{6}$. Por ello, es plausible defender que las inferencias, predicciones u opiniones sobre un individuo pueden calificarse como datos personales si se utilizan en un ámbito de personalización de decisiones (WACHTER, S. - MITTELSTADT, 2019, 541). La finalidad de evaluación y la adopción de decisiones de personalización basadas en ellas justificarían este estatus.

Otro problema relativo a las inferencias es si pueden llegar a constituir datos personales sensibles cubiertos por el art. 9 RGPD, a pesar de ser obtenidas mediante datos inicialmente no sensibles (FINCK, M., 2021, 101-102).

La naturaleza subjetiva de estas inferencias empleadas en la elaboración de perfiles y la personalización dificulta el ejercicio de derechos de los afectados. Rectificar un juicio de valor -que, de entrada, no es susceptible de ser falso o verdadero- no puede funcionar igual que la rectificación de un hecho objetivo. Por otra parte, las inferencias y

\footnotetext{
${ }^{6}$ STJUE de 20 de diciembre de 2017, C-434/16, Peter Nowak c. Data Protection Commissioner. Cfr. STJUE de 17 de julio de 2014, C-141/12 y C-372/12, YS, M y S c. Minister voor Immigratie, Integratie en Asiel.
} 
correlaciones derivadas de estas formas de tratamiento resultan de la evaluación y combinación de datos personales de un sujeto con los de otros individuos o con patrones generales agregados obtenidos de la observación de otros individuos. Ello también dificulta muy mucho el ejercicio de derechos como el de supresión y objeción al tratamiento: en el estado actual de la tecnología un ejercicio efectivo individual de estos derechos podría comportar que el algoritmo utilizado para la personalización deba dejarse de utilizarse y debiera volver a activarse con una nueva base de datos para entrenarlo. Los costes económicos, laborales y medioambientales que ello generaría sugieren que el arsenal de derechos previsto en el RGPD es más bien impracticable en estos casos (FINCK, M., 2021, 99-100).

\section{Conclusiones}

La personalización en los contratos con consumidores ha permitido prestar servicios de forma más ajustada a las preferencias e intereses de aquellos, pero también ha generado algunos efectos perniciosos. Hasta cierto punto, la competencia en el mercado, el reproche social o la propia tecnología pueden mitigar algunos de estos problemas, pero donde no lleguen o no puedan actuar eficazmente, una solución jurídica resultará más plausible.

Puesto que la personalización comporta tratamientos masivos de datos personales y, en especial, la elaboración de perfiles de consumidores, el derecho de protección de datos constituye uno de los principales sectores del ordenamiento en el cual examinar las soluciones jurídicas disponibles. Mas el régimen previsto actualmente en el RGPD resulta insuficiente en muchos aspectos para hacer frente a muchos de los problemas que plantea la personalización. Como se ha señalado, las normas específicas relativas a la elaboración de perfiles previstas en el art. 22 RGPD no serán aplicables en muchos supuestos de personalización; el estatus jurídicos de las inferencias y predicciones de las que se nutre el análisis de datos para adoptar decisiones de personalización es ambiguo y limita la fuerza del ejercicio de derechos individuales, tales como el derecho de acceso, rectificación y supresión; o los requisitos sobre el consentimiento y la información a suministrar son insuficientes para proporcionar un conocimiento efectivo a los consumidores acerca de cómo sus características personales determinan varios elementos de los contratos en los que participan. El derecho de protección de datos no está bien equipado para asegurar un excedente contractual a los consumidores o para combatir 
prácticas de manipulación de sus preferencias. No es claro tampoco si los principios de licitud y lealtad (art. 5.1 RGPD) justificarían que el derecho de protección de datos asumiera estas funciones. Por ello, el régimen previsto en el RGPD debe complementarse con otros, como, por ejemplo, el derecho de defensa de la competencia (MAGGIOLINO, M., 2017), el derecho represor de la competencia desleal (LAUX, J. - WACHTER, S. MITTELSTADT, B., 2021) y el derecho de consumo (HELBERGER, N. ZUIDERVEEN BORGESIUS, F. - REYNA, A., 2017).

\section{Referencias bibliográficas}

ALFARO, J. (2017), "Precios personalizados y discriminación”, Almacén de Derecho, 5.12.2017 (disponible en https://almacendederecho.org/precios-personalizadosdiscriminacion; consultado en 15.8.2021).

BAR-GILL, O. (2019), “Algorithmic Price Discrimination When Demand Is a Function of Both Preferences and (Mis)perceptions", University of Chicago Law Review, 86, 2, pp. 217-254.

BAROCAS, S. - LEVI, K. (2020), "Privacy Dependencies", Washington Law Review, 95, 2, pp. 555-616.

BEN-SHAHAR, O. - SCHNEIDER, C. (2014), More Than You Wanted to Know: The Failure of Mandated Disclosure, Princeton/Oxford: Princeton University Press.

BINNS, R. - VEALE, M. (2021), "Is That Your Final Decision? Multi-Stage Profiling, Selective Effects, and Article 22 of the GDPR", Internet Data Privacy Law (en prensa) (disponible en: https://doi.org/10.31235/osf.io/7mq6z).

BÜCHI, M. - FOSCH VILLARONGA, E., et al (2019), “Chilling Effects of Profiling Activities: Mapping the Issues" (disponible en: https://ssrn.com/abstract=3379275).

CALO, R. (2014), "Digital market manipulation", George Washington Law Review 82, 4, pp. 995-1051. 
CASEY, B. - FARHANGI, A. - VOGL, R. (2019), "Rethinking Explainable Machines: The GDPR's 'Right to Explanation' Debate and the Rise of Algorithmic Audits in Enterprise”, Berkeley Technology Law Journal, 34, 1, pp. 143-188.

CITRON, D. - PASQUALE, F. (2014), “The scored society: Due process for automated predictions", Washington Law Review, 89, 1, pp. 1-33.

COMITÉ EUROPEO DE PROTECCIÓN DE DATOS (2019), Directrices 2/2019 sobre el tratamiento de datos personales en virtud del artículo 6, apartado 1, letra b), del RGPD en el contexto de la prestación de servicios en línea a los interesados (versión 2.0 de 8.10.2019).

FINCK, M. (2021), "Hidden Personal Insights and Entangled in the Algorithmic Model: The Limits of the GDPR in the Personalisation Context", en KOHL, U. - EISLER J. (Eds.), Data-Driven Personalisation in Markets, Politics and Law, Cambridge, Cambridge University Press, pp. 95-107.

GRUPO DE TRABAJO SOBRE PROTECCIÓN DE DATOS DEL ARTÍCULO 29 (2017), Directrices sobre decisiones individuales automatizadas y elaboración de perfiles a los efectos del Reglamento 2016/679 (adoptadas el 3.102017 - revisadas por última vez y adoptadas el 6.2.2018).

HELBERGER, N. - ZUIDERVEEN BORGESIUS, F. - REYNA, A. (2017), “The perfect match? A closer look at the relationship between EU consumer law and data protection law", Common Market Law Review 54, 5, pp. 1427-1465.

KLEINBERG, J.- LUDWIG, S. et al (2018), “Discrimination in the age of algorithm", Journal of Legal Analysis, 10, pp. 113-174.

LAUX, J. - WACHTER, S. - MITTELSTADT, B. (2021), "Neutralizing Online Behavioural Advertising Algorithmic Targeting with Market Power as an Unfair Commercial Practice", Common Market Law Review, 58, 3, pp. 719-750. 
LEVMORE, S. - FAGAN, F. (2018), "The End of Bargaining in the Digital Age", Cornell Law Review, 103, 6, pp. 1469-1526.

MAGGiOLINO, M. (2017), "Personalized Prices in European Competition Law", Bocconi Legal Studies Research Paper No. 2984840 (disponible en: https://ssrn.com/abstract=2984840).

MILLER, A. (2014), "What Do We Worry about When We Worry about Price Discrimination? The Law and Ethics of Using Personal Information for Pricing”, Journal of Technology Law and Policy, 19, pp. 41-104.

PASQUALE, F. (2016), The Black Box Society. The Secret Algorithms That Control Money and Information, Cambridge: Harvard University Press.

RUBÍ PUIG, A. (2015), "Principio salva rerum substantia y agotamiento de derechos de distribución”, InDret, 3, pp. 1-45 (www.indret.com).

RUBÍ PUIG, A. (2019), "Problemas de coordinación y compatibilidad entre la acción indemnizatoria del artículo 82 del Reglamento General de Protección de Datos y otras acciones en derecho español”, Derecho Privado y Constitución, 34, pp. 197-232.

SARTOR, G.- LAGIOIA, F. - GALLI, F. (2021), "Regulating targeted and behavioural advertising in digital services. How to ensure users' informed consent”, Study requested by the JURI committee. PE 694.680 - July 2021 (disponible en https://www.europarl.europa.eu/RegData/etudes/IDAN/2021/694680/IPOL_STU(2021) 694680_EN.pdf. Consultado en 15.8.2021).

SWEENEY, L. (2013), “Discrimination in Online Ad Delivery”, Queue, 11, 3, pp. 1029.

VARIAN, H. (2014), Intermediate Microeconomics: A Modern Approach, $9^{\mathrm{a}}$ ed., New York/Londres: W.W. Norton. 
VEALE, M. - ZUIDERVEEN BORGESIUS, F. (2021), “Adtech and Real-Time Bidding under European Data Protection Law", German Law Journal (en prensa) (disponible en https://doi.org/10.31235/osf.io/wg8fq).

VILASAU, M. (2020), "La realización de perfiles y la salvaguardia de los derechos y libertades del afectado", en CERRILLO, A. - PEGUERA, M. (coords.), Retos jurídicos de la inteligencia artificial, Cizur Menor, Thomson Aranzadi, pp. 181-202.

WACHTER, S. (2020), “Affinity Profiling and Discrimination by Association in Online Behavioural Advertising”, Berkeley Technology Law Journal, 35, 2, pp. 367-430.

WACHTER, S. - MITTELSTADT, B. (2019), “A Right to Reasonable Inferences: ReThinking Data Protection Law in the Age of Big Data and AI", Columbia Business Law Review, 2019, 2, pp.494-620.

WAGNER, G. - EIDENMÜLLER, H. (2019), "Down by Algorithms? Siphoning Rents, Exploiting Biases and Shaping Preferences - The Dark Side of Personalized Transactions", University of Chicago Law Review, 86, 2, pp. 581-609.

WILLIS, L. (2020), "Deception by Design”, Harvard Journal of Law and Technology, 34, pp. 115-190.

ZUIDERVEEN BORGESIUS, F. - POORT, J. (2017), “Online Price Discrimination and EU Data Privacy Law”, Journal of Consumer Policy, 40, pp. 347-366.

ZUIDERVEEN BORGESIUS, F. - TRILLING, D. et al (2016), "Should we worry about filter bubbles?”, Internet Policy Review, 5, 1, pp. 1-16. 